cigarette smoking (based on smoking rates in a cross-sectional survey) did not influence the estimates.

Conclusions While the results of this analysis did not reach statistical significance, they provide evidence supporting previous studies showing a risk of COPD associated with MWF exposure. The hazards presented are likely to be underestimates of the true association between COPD and MWF, due to the healthy worker effect.

\section{CANCER, MORTALITY AND ACUTE MYOCARDIAL INFARCTION IN WORKERS EXPOSED TO RESPIRABLE CRYSTALLINE SILICA DUST AT A SWEDISH PORCELAIN FACTORY}

1,2Pernilla Wiebert, ${ }^{2}$ Magnus Alderling, ${ }^{3}$ Magnus Svartengren, 1,2Per Gustavsson, ${ }^{1}$ Nils Plato. 'Karolinska Institutet, Stockholm, Sweden; ${ }^{2}$ Stockholm County Council, Stockholm, Sweden; ${ }^{3}$ Department of Medical Sciences, Uppsala, Sweden

10.1136/oemed-2014-102362.96

Objectives Exposure to silica dust is a health hazard in the ceramic industry. We studied cancer, mortality and acute myocardial infarction (AMI) among workers at a Swedish porcelain factory.

Method Annual average of exposure levels were estimated from 436 personal measurements of respirable crystalline silica dust (RCS) from 1971-2006. We investigated mortality, incidence of cancer, and first time event of AMI in men and women employed for at least one year at the factory in 1958-2009. We also studied the effect of latency, duration and cumulative exposure.

Results RCS-levels among highly exposed workers were five times higher than the OEL and ten times higher in the early 1970 s as in 2000.

We found a non-significant elevated risk for lung cancer, (SIR 1.39 ; $95 \%$ CI $0.79-2.25$ ) and a significant elevated risk of squamous cell carcinoma in men (SIR 2.37; 1.02-4.66).

Mortality from respiratory diseases was increased (SMR 1.75; $1.22-2.44$ ), especially in men (SMR $1.86 ; 1.22-2.70$ ). Among women, the risk for mortality from diseases of the circulatory system and incidence of AMI was elevated but not statistically significant. We found no dose-response relationship. There were eight cases of silicosis, and seven appeared with more than 30 years latency.

Conclusions The increased risk for lung cancer and mortality from respiratory diseases was expected in view of the well-documented harmful effects of RCS. The tendency among women for increased mortality from diseases of the circulatory system and an increase in the incidence of AMI should be investigated in further studies.

\section{PERCEIVED WORKPLACE DISCRIMINATION AND SELF RATED HEALTH IN THE CHILEAN WORKFORCE}

Junhee Cho, Devan Hawkins, Laura Kernan, Manuel Cifuentes. University of Massachusetts Lowell, Work Enviroment, Lowell, USA

10.1136/oemed-2014-102362.97

Objectives Increased research shows that perceived discrimination adversely affects physical and psychological health. Even though discrimination or concealed racism is an important characteristic of the Chilean society, which can be confirmed historically, it is not perceived as an important social problem for mainstream Chile. This paper aims to estimate the prevalence rate of workplace perceived discriminatory experience (WPDE) and its association with self-rated health status in the Chilean workforce

Method Data from the first national survey on employment, work, and health in Chile. Study population of 9720 selected by multistage random sampling drawn to be representative of the entire working population. Study participants were asked about their WPDE (multiple questions) and general self rated health status (one question). Adjusting by demographic and socioeconomic factors, multivariable Poisson-log generalised linear mixed models were used to estimate the association between WPED and self-rated health.

Results Approximately 17\% reported being a victim of WPDE. Age, income, education, and minority (nine ethnicities) were strongly associated with WDPE. Female workers showed higher rate $(19.6 \%)$ of WPDE than male workers (15.6\%). After simultaneously controlling for potential confounders, WPDE was positively associated with poor self-rated health $(\mathrm{PR}=2.12, \mathrm{CI}=$ 1.46-3.05).

Conclusions There is positive association between WPDE and poor self-rated health in Chile. These results may be used to emphasise the importance of enacting preventive and protective workplace discrimination policies. Further research is required to study the causal mechanism of the link and best preventive and protective measures.

\section{ACUTE INFLAMMATORY RESPONSE TO SECONDHAND SMOKE EXPOSURE AMONG NON-SMOKING CONSTRUCTION WORKERS: A REPEATED MEASURES STUDY}

${ }^{1} J e n n i f e r$ Bruno Garza, ${ }^{2}$ Jinming Zhang, ${ }^{2}$ Shona Fang, ${ }^{2}$ Jason Wong, ${ }^{2}$ David Christiani, ${ }^{1}$ Jennifer Cavallari. 'University of Connecticut Health Center, Farmington, CT, USA; ${ }^{2}$ Harvard School of Public Health, Boston, MA, USA

\subsection{6/oemed-2014-102362.98}

Objectives This study aimed to characterise the cardiovascular inflammatory response to secondhand smoke (SHS) exposure among non-smoking construction workers.

Method Non-smoking workers $(\mathrm{n}=27)$ were recruited from a local union and monitored inside a union hall while exposed to SHS over approximately $6 \mathrm{~h}$. Using a repeated measures study design, blood samples were taken before SHS exposure (baseline), immediately following SHS exposure (post) and the morning following SHS exposure (next morning). Inflammatory markers including acute phase proteins (SAA, CRP), adhesion molecules (s-ICAM, s-VCAM), and inflammatory cytokines (IL1, IL-2, IL-6, IL-8, IL-10, TNF-alpha, VEGF) were analysed. Linear mixed effects regression models were used to examine within-person changes in inflammatory markers at post and next morning compared to baseline. Exposure-response relationships with TWA PM2.5 were also examined using mixed effects models. All models were adjusted for age, BMI and circadian variation.

Results There was a decrease in SAA (baseline $=2322 \mathrm{ng} / \mathrm{ml}$, post $=1949 \mathrm{ng} / \mathrm{ml}, \mathrm{p}=0.04$ ) and TNF-alpha (baseline $=9.6$ $\mathrm{pg} / \mathrm{ml}$, post $=8.4 \mathrm{pg} / \mathrm{ml}, \mathrm{p}<0.01)$ post exposure, as compared to baseline. There was a decrease in IL-10 (baseline $=5.9 \mathrm{pg} / \mathrm{ml}$, next morning $=6.5 \mathrm{pg} / \mathrm{ml}, \mathrm{p}<0.01)$ next morning compared to 